\title{
THE TRIANGULAR THEOREM OF EIGHT AND REPRESENTATION BY QUADRATIC POLYNOMIALS
}

\author{
WIEB BOSMA AND BEN KANE
}

(Communicated by Kathrin Bringmann)

\begin{abstract}
We investigate here the representability of integers as sums of triangular numbers, where the $n$-th triangular number is given by $T_{n}=$ $n(n+1) / 2$. In particular, we show that $f\left(x_{1}, x_{2}, \ldots, x_{k}\right)=b_{1} T_{x_{1}}+\cdots+b_{k} T_{x_{k}}$, for fixed positive integers $b_{1}, b_{2}, \ldots, b_{k}$, represents every nonnegative integer if and only if it represents $1,2,4,5$, and 8 . Moreover, if 'cross-terms' are allowed in $f$, we show that no finite set of positive integers can play an analogous role, in turn showing that there is no overarching finiteness theorem which generalizes the statement from positive definite quadratic forms to totally positive quadratic polynomials.
\end{abstract}

\section{INTRODUCTION}

In 1638 Fermat claimed that every number is a sum of at most three triangular numbers, four square numbers, and in general $k$ polygonal numbers of order $k$. The $n$-th polygonal number of order $k$ is $\frac{(k-2) n^{2}-(k-4) n}{2}$, so the $n$-th triangular number is $T_{n}:=\frac{n(n+1)}{2}$, where we include $T_{0}=0$ for simplicity. The claim for four squares was shown by Lagrange.

Theorem (Lagrange, 1770). Every positive integer is the sum of four squares.

Gauss wrote "Eureka, $\triangle+\triangle+\triangle=n$ " in his mathematical diary on July 10, 1796.

Theorem (Gauss, 1796). Every positive integer is the sum of three triangular numbers.

The first proof of the full assertion of Fermat was given by Cauchy in 1813 [3]; cf. [12.

For a more complete history of related questions about sums of figurate numbers and some new results, see Duke's survey paper 8 .

The current paper concerns questions of representability of integers by quadratic polynomials. If $f=f(x)=f\left(x_{1}, x_{2}, \ldots, x_{k}\right)$ is a rational polynomial in $k$ variables, it represents the integer $n$ if there exist integers $n_{i}$ such that $n=f\left(n_{1}, n_{2}, \ldots, n_{k}\right)$, and it oddly represents the integer $n$ if there exist odd integers $n_{i}$ such that $f\left(n_{1}, n_{2}, \ldots, n_{k}\right)=n$. If $f$ represents every element of a set $\mathcal{Z}$ of integers, it is said to represent $\mathcal{Z}$.

Received by the editors December 6, 2010 and, in revised form, August 4, 2011 and August 25, 2011.

2010 Mathematics Subject Classification. Primary 11E25, 11E20, $11 \mathrm{E} 45$.

Key words and phrases. Triangular numbers, quadratic forms, sums of odd squares. 
If we let $S=S_{x}$ be the square polynomial $x^{2}$, and let $T=T_{x}$ denote the triangular polynomial $\left(x^{2}+x\right) / 2$, the theorems of Lagrange and Gauss state that the positive integers are represented by $S_{w}+S_{x}+S_{y}+S_{z}$ and by $T_{x}+T_{y}+T_{z}$.

In 1917, Ramanujan extended the question about four squares to ask for which choices of quadruples $b=\left(b_{1}, b_{2}, b_{3}, b_{4}\right)$ of integers the form $b_{1} S_{w}+b_{2} S_{x}+b_{3} S_{y}+b_{4} S_{z}$ represents every positive integer; we shall refer to these as universal diagonal forms. He gave a list of 55 choices of $b$ which he claimed to be the complete list of universal quarternary diagonal forms; 54 of them turned out to be universal, and this list is complete, as proven by Dickson 7 .

Recently, Conway and Schneeberger proved in unpublished work a nice classification for universal positive definite quadratic forms whose corresponding matrices have integer entries. This answers the question of representability by positive definite homogeneous quadratic polynomials with even off-diagonal coefficients.

Theorem (Conway-Schneeberger). A positive definite quadratic form $Q(x)=x^{t} A x$, where $A$ is a positive symmetric matrix with integer coefficients, represents every positive integer if and only if it represents the integers 1,2,3, 5, 6, 7, 10,14, and 15.

Bhargava gave a simpler proof of the Conway-Schneeberger 15-Theorem in [1] and showed more generally that representability of any $\mathcal{Z}$ by such a form can always be checked on a finite subset $\mathcal{Y}$. In addition, he exhibited $\mathcal{Y}$ for $\mathcal{Z}$ consisting of all odd integers and for $\mathcal{Z}$ consisting of all primes.

More recently, Bhargava and Hanke [2] have shown the 290-Theorem, providing the necessary set (the largest element of which is 290) for universal forms when the corresponding matrix is half integral, that is, for totally positive integer quadratic forms.

In 1863, Liouville [11 proved the following generalization of Gauss's theorem, similar to Ramanujan's generalization of Lagrange's Four Squares Theorem.

Theorem (Liouville). Let $a, b, c$ be positive integers with $a \leq b \leq c$. Then every positive integer is represented by $a T_{x}+b T_{y}+c T_{z}$ if and only if $(a, b, c)$ is one of the following:

$$
(1,1,1),(1,1,2),(1,1,4),(1,1,5),(1,2,2),(1,2,3),(1,2,4) .
$$

We will first prove a finiteness theorem similar to the results of the ConwaySchneeberger 15-Theorem or the Bhargava-Hanke 290-Theorem for sums of triangular numbers.

Theorem 1.1. If $b_{1}, \ldots, b_{k}$ is a sequence of positive integers, then $\sum_{i=1}^{k} b_{i} T_{x_{i}}$ represents every nonnegative integer if and only if it represents $1,2,4,5$, and 8.

Since $8 T_{x}=(2 x+1)^{2}-1$, clearly $\sum_{i=1}^{k} b_{i} T_{n_{i}}=n$ if and only if $\sum_{i=1}^{k} b_{i}\left(2 n_{i}+1\right)^{2}=$ $8 n+\sum_{i=1}^{k} b_{i}$. Hence there is a close correspondence between representability by triangular polynomials and odd representability by diagonal quadratic forms.

Corollary 1.2. If $b_{1}, \ldots, b_{k}$ is a sequence of positive integers with sum $B$, then $\sum_{i=1}^{k} b_{i} x_{i}^{2}$ oddly represents every integer of the form $8 n+B$ with $n \geq 0$ if and only if it oddly represents $8+B, 16+B, 32+B, 40+B$, and $64+B$.

It is not so difficult to establish Theorem 1.1] with the escalator techniques of Bhargava (and Liouville). We will prove a stronger statement in Section 2: if the integers $1,2,4,5$, and 8 are represented by the triangular form, then $n$ is represented very many times unless $n+1$ has high 3 -divisibility. 
We now turn to more general quadratic polynomials. Let $f$ be a quadratic polynomial in $\mathbb{Q}\left[x_{1}, x_{2}, \ldots, x_{k}\right]$; then $f$ is a normalized totally positive quadratic polynomial if the image of $\mathbb{Z}^{k}$ under $f$ consists of nonnegative integers, while $f(x)=$ 0 for some $x \in \mathbb{Z}^{k}$. Note that clearly $S_{x}=x^{2}$ is normalized totally positive, as is $T_{x}: T_{0}=0, T_{1}=1, T_{2}=3$ are the first of the increasing sequence of triangular numbers, and $T_{-m}=T_{m-1}$ for positive $m$.

It turns out that no finiteness theorem will hold in general for normalized totally positive quadratic polynomials, and moreover that checking no proper subset will suffice.

Proposition 1.3. Let $\mathcal{Z}$ be a subset of the positive integers. For every proper subset $\mathcal{Y} \subsetneq \mathcal{Z}$ there exists a normalized totally positive quadratic polynomial that represents $\mathcal{Y}$ but does not represent $\mathcal{Z}$.

Proposition 1.3 will follow directly from the corresponding result for triangular sums with cross terms. This class corresponds to integral quadratic forms with even off-diagonal terms, just as the ordinary triangular sums correspond to diagonal quadratic forms. We refer to Section 3 for a precise definition of this subclass of quadratic polynomials.

In Section 4 we construct a 'norm' $m$ on this class that restores finite representability.

Theorem 1.4. Fix an integer $m$ and a subset $\mathcal{Z}$ of the positive integers. Then there is a finite subset $\mathcal{Y}_{m} \subset \mathcal{Z}$, depending only on $m$ and $\mathcal{Z}$, such that every triangular sum $t$ with cross terms satisfying $m(t) \leq m$ represents $\mathcal{Z}$ if and only if it represents $\mathcal{Y}_{m}$.

Moreover, for $\mathcal{Z}$ equal to the positive integers, we find that $\max \mathcal{Y}_{m} \gg m^{2}$.

It may be of interest to investigate the growth of $\max \mathcal{Y}_{m}$; see Remark 4.3.

\section{THEOREM OF EIGHT}

For background information on quadratic forms and genus theory, a good source is [9. We prove Theorem 2.1, by using a standard argument to show that the theorem is equivalent to a statement about (diagonal) quadratic forms, and then prove the corresponding result for quadratic forms. We will only need some elementary results about quadratic forms and a theorem of Siegel to show the desired result. Theorem 1.1 and Corollary 1.2 follow immediately.

We will first introduce some useful notation and definitions. We abbreviate $t(x)=t\left(x_{1}, x_{2}, \ldots, x_{k}\right):=\sum b_{i} T_{x_{i}}$, and call it a triangular sum. For a vector $b$ of length $k$ we define the generating function

$$
F(q):=F_{b}(q):=\sum_{x \in \mathbb{Z}^{k}} q^{t(x)}=\sum_{n=0}^{\infty} s_{b}(n) q^{n},
$$

where $s_{b}(n)$ is the number of solutions to $t(x)=n$. We will omit the subscript of $s_{b}(n)$ when it is clear from the context. We will furthermore use $r(n)$ to denote the number of representations of $n$ by the corresponding (diagonal) quadratic form $\sum b_{i} x_{i}^{2}$ and $r_{o}(n)$ to denote the number of those representations with all $x_{i}$ odd. For ease of notation, we will denote the triangular sum corresponding to $b$ with $\left[b_{1}, b_{2}, \ldots, b_{k}\right]$ and the corresponding quadratic form by $\left(b_{1}, \ldots, b_{k}\right)$. 
The Hurwitz class number for the imaginary quadratic order of discriminant $D<$ 0 will play an important role in our analysis below. We recall the definition here. For a negative discriminant $D$, the Hurwitz class number $H(D)$ is the weighted number of equivalence classes of, not necessarily primitive, positive definite binary quadratic forms of discriminant $D$, where the weights are 1 except for classes of forms equivalent to a multiple of $\left(x^{2}+y^{2}\right)$, which are counted with weight $\frac{1}{2}$, and for classes of forms equivalent to a multiple of $\left(x^{2}+x y+y^{2}\right)$, which are counted with weight $\frac{1}{3}$. Every quadratic form of discriminant $D$ is a multiple of a primitive form of discriminant $D^{\prime}=D / f^{2}$, and the weights are reciprocal to $w\left(D^{\prime}\right) / 2$, half the number of units in the unique order of discriminant $D^{\prime}$, or, accordingly, to half the number of representations of the integer 1 by the primitive form. The usual class numbers $h(D)$ are hence related to the Hurwitz class number by

$$
H(D)=\sum_{f^{2} \mid D} \frac{h\left(\frac{D}{f^{2}}\right)}{\frac{1}{2} w\left(\frac{D}{f^{2}}\right)} .
$$

For an integer $n$, we will set $a_{n}:=\frac{v_{3}(n+1)}{\log _{3}(n+1)}$, so that $3^{v_{3}(n+1)}=(n+1)^{a_{n}}$ gives the 3 -part of $n+1$ as a power of $n+1$.

Theorem 2.1. For $\epsilon>0$, there is an absolute constant $c_{\epsilon}$ such that if the triangular sum $t(x)$ represents $1,2,4,5$, and 8 , then $t(x)$ represents every nonnegative integer $n$ at least $\min \left\{c_{\epsilon} n^{\frac{1}{2}-\epsilon}, 16 n^{1-a_{n}}\right\}$ times. In particular, if $n$ is sufficiently large and $a_{n}<\frac{1}{2}$, then $t(x)$ represents $n$ at least $c_{\epsilon} n^{\frac{1}{2}-\epsilon}$ times.

Proof. We proceed with escalator lattices as in [1]. Without loss of generality we have $b_{1} \leq b_{2} \leq \cdots \leq b_{k}$. Fixing $b=\left[b_{1}, \ldots, b_{k-1}\right]$, we will escalate to $\left[b_{1}, \ldots, b_{k}\right]$ by making all possible choices of $b_{k} \geq b_{k-1}$ for which it is possible to represent the next largest integer not already represented. We will then develop an escalator tree by forming an edge between $b$ and $\left[b_{1}, \ldots, b_{k}\right]$, with $\emptyset$ as the root. If $\sum_{i} b_{i} T_{x_{i}}$ represents every integer, then $b$ will be a leaf of our tree.

Since $s(1)>0$, it follows that $b_{1}=1$. We need $s(2)>0$, so $b_{2}=1$ or $b_{2}=2$. If $b_{2}=1$, then we need $s(5)>0$, so $1 \leq b_{3} \leq 5$. For $b_{3}=3$, we need $s(8)>0$, so $3 \leq b_{4} \leq 8$. Likewise, if $b_{2}=2$, then $2 \leq b_{3} \leq 4$. Therefore, if $s(n)>0$ for every $n$, then we must have one of the above choices of $b_{i}$ as a sublattice. By showing that each of these choices of $b_{i}$ satisfies $s(n)>0$ for every $n$, we will see that this condition is both necessary and sufficient.

All of the cases other than $[1,1,3, k]$ with $3 \leq k \leq 8$ are covered by Liouville's Theorem. However, to obtain the more precise version given in Theorem 2.1 we will use quadratic form genus theory.

One sees easily that

$$
q^{\sum_{i=1}^{k} b_{i}} F\left(q^{8}\right)=\sum_{x} q^{\sum_{i=1}^{k} b_{i}\left(2 x_{i}+1\right)^{2}}
$$

so that $s(n)=r_{o}\left(8 n-\sum_{i=1}^{k} b_{i}\right)$. For the forms $b=[1,1,1],[1,1,4],[1,1,5]$, $[1,2,2]$, and $[1,2,4]$, congruence conditions modulo 8 imply that

$$
r_{o}\left(8 n-\sum_{i=1}^{k} b_{i}\right)=r\left(8 n-\sum_{i=1}^{k} b_{i}\right) .
$$


Moreover, for each of these choices of $b,\left(b_{1}, b_{2}, b_{3}\right)$ is a genus 1 quadratic form. Therefore, extending the classification of Jones [9, Theorem 86] to primitive representations when the integer is not squarefree, $s_{[1,1,1]}(n)=24 H(-(8 n+3))$, $s_{[1,1,4]}(n)=4 H(-4(8 n+6)), s_{[1,2,2]}(n)=4 H(-4(8 n+5))$, and $s_{[1,2,4]}(n)=$ $2 H(-8(8 n+7))$.

For $[1,1,5]$ we must be slightly more careful since 5 divides the discriminant. We will explain in some detail how to deal with this complication and then will henceforth ignore this difficulty when it arises. For $5 \nmid 8 n+7$ we have $s_{[1,1,5]}(n)=$ $4 H(-5(8 n+7))$. Hence the only difficulty occurs with high divisibility by 5 . For $p \neq 5$ the local densities are equal to those for bounded divisibility. Thus, entirely analogously to the result of Jones we have $s_{[1,1,5]}(n)=c_{n} H(-5(8 n+7))$ for some constant $c_{n}>0$ which only depends 5 -adically on $8 n+7$. We calculate the cases $v_{5}(8 n+7) \leq 3$ by hand. Denote 5 -primitive representations of $m$ (i.e., $5 \nmid \operatorname{gcd}(x, y, z))$ by $r^{*}(m)$. Checking locally, for $5^{2} \mid m:=8 n+7$, we will obtain the result inductively by showing $\frac{r^{*}(25 m)}{r^{*}(m)}=\frac{h(25 m) / u(25 m)}{h(m) / u(m)}$ and then summing to get $r(m) \geq 4 H(-5 m)$. But, since $5 \mid m$, we have $\frac{h(25 m) / u(25 m)}{h(m) / u(m)}=5$ by the class number formula (see [5, Corollary 7.28, page 148]) so that this is a quick local check at the prime 5 .

Our proofs for $[1,1,2],[1,2,3]$, and $[1,1,3]$ will be essentially identical. For $[1,1,2]$, we note that if $x^{2}+y^{2}+2 z^{2}=8 n+4$ has a solution with $x, y$, and $z$ not all odd, then taking each side modulo 8 leads us to the conclusion that $x, y$, and $z$ must all be even. Therefore, the solutions without $x, y$, and $z$ odd correspond to solutions of

$$
4 x^{2}+4 y^{2}+8 z^{2}=8 n+4, \quad \text { that is, of } x^{2}+y^{2}+2 z^{2}=2 n+1 .
$$

Using Siegel's theorem to compare the local density at 2 , we see that the average of the number of representations over the genus is three times as large for $8 n+4$ as $2 n+1$. However, $(1,1,2)$ is again a genus 1 quadratic form, so $r(8 n+4)=3 r(2 n+1)$, and hence $s_{[1,1,2]}(n)=r_{o}(8 n+4)=r(8 n+4)-r(2 n+1)=2 r(2 n+1)$. Thus by Theorem 86 of Jones [9] we have $s_{[1,1,2]}(n)=8 H(-8(2 n+1))$. Similar arguments show that

$$
\begin{aligned}
s_{[1,2,3]}(n)=r_{o,(1,2,3)}(8 n & +6)=r_{(1,2,3)}(8 n+6)-r_{(4,2,12)}(8 n+6) \\
& =r_{(1,2,3)}(8 n+6)-r_{(1,2,6)}(4 n+3)=2 r_{(1,2,6)}(4 n+3) .
\end{aligned}
$$

Similar to the case $[1,1,5]$, we have $s_{[1,2,3]}(n) \geq 2 H(-12(4 n+3))$.

For $[1,1,3]$ we see analogously that

$$
s_{[1,1,3]}(n)=r_{o,(1,1,3)}(8 n+5)=r_{(1,1,3)}(8 n+5)-r_{(1,1,12)}(8 n+5)=r_{(1,1,12)}(8 n+5) \text {, }
$$

and again $(1,1,12)$ is genus 1 . We conclude in the case $3 \nmid(8 n+5)$ that we have $s_{[1,1,3]}(n)=4 H(-3(8 n+5))$, and we may henceforth assume that $3 \mid 8 n+5$ (i.e. $n \equiv 2(\bmod 3))$. Local conditions imply that $3^{2 j+1}(3 \ell+2)$ is not represented by $(1,1,3)$, so we have escalated to $[1,1,3, k]$ for $k$ such that $3 \leq k \leq 8$. For $3 \nmid k$, by choosing $x_{4}=1$ we have $s_{[1,1,3, k]}(n) \geq 4 H(-3(8(n-k)+5))$ since $3 \nmid 8(n-k)+5$. For $k=3$ we have

$$
s_{[1,1,3,3]}(n)=r_{(1,1,3,3)}(8(n+1))+r_{(4,4,12,12)}(8(n+1))-2 r_{(1,3,3,4)}(8(n+1)) .
$$

Denoting the usual $d$-th degeneracy $V$-operator by $V(d)$ and the usual $U$-operator by $U(d)$ (cf. p. 28 of [13]), one may write the difference of the $\theta$-series $\sum_{n} r(8 n) q^{n}$ 
for these quadratic forms as

$$
\theta_{(1,1,3,3)}\left|U(8)+\theta_{(1,1,3,3)}\right| V(4)\left|U(8)-2 \theta_{(1,3,3,4)}\right| U(8) .
$$

It is easy to conclude that the generating function $q F(z)=\sum_{n} s_{[1,1,3,3]}(n) q^{n+1}$, with $q=e^{2 \pi i z}$, is a weight 2 modular form of level 48. Using Sturm's bound [15] and checking the first 16 coefficients reveals that $q F(z)=16 \frac{\eta(2 z)^{4} \eta(6 z)^{4}}{\eta(z)^{2} \eta(3 z)^{2}}$. The coefficients are multiplicative, so that if we have the factorization $n+1=2^{e} 3^{f} \prod_{p>3} p^{e_{p}}$, then

$$
s_{[1,1,3,3]}(n)=2^{e+4} \prod_{p>3} \frac{p^{e_{p}+1}-1}{p-1} \geq 16 \frac{n+1}{3^{f}}=16(n+1)^{1-a_{n}} .
$$

Finally, for $k=6$ we check $n<10$ by hand and then note that

$$
s_{[1,3,6]}(n)=r_{(1,3,6)}(8 n+10)-r_{(2,3,6)}(4 n+5),
$$

while both $(1,3,6)$ and $(2,3,6)$ are genus 1 . Hence for $n \not \equiv 2(\bmod 3)$ we have $s_{[1,3,6]}(n) \geq 2 H(-4(4 n+5))$. We then take the remaining variable $x_{4}=1$ to obtain for $n \equiv 2(\bmod 3)$ that $s_{[1,1,3,6]}(n) \geq 2 H(-4(4(n-1)+5))$, since $n-1 \not \equiv 2(\bmod 3)$.

Having seen that each of our choices of $b$ is indeed a leaf to the tree, we conclude that representing the integers $1,2,4,5$, and 8 suffices.

Remark 2.2. The constant $c_{\epsilon}$ in Theorem 2.1 is ineffective because it relies on Siegel's lower bound for the class number, but the bound of $c_{\epsilon} n^{\frac{1}{2}-\epsilon}$ may be replaced with the minimum of finitely many choices of a constant times a Hurwitz class number of a certain imaginary quadratic order whose discriminant is linear in $n$.

We have the following example. In this example, instead of considering $s_{b}(n)$, we normalize the number of representations by

$$
s_{b}^{\prime}(n):=\frac{s_{b}(n)}{2^{k}},
$$

where $k$ is the length of the sequence $b$. This normalization is made so that $T_{x_{i}}=T_{-x_{i}-1}$ appears exactly once and in particular implies that 0 is represented precisely once. Using this normalization and the explicit bound in terms of the Hurwitz class number, we obtain for instance that if $1,2,4,5$, and 8 are represented, then the integer 195727301431 is represented at least 270390 times and the integer 48291403767737750 is necessarily represented at least 90542761 times (here $a_{n} \approx 0.364$ ), while the integer $50031545098999706=3^{35}-1$ is only necessarily represented once. All of the bounds listed in these examples are sharp (i.e., there exists a triangular sum representing 1,2,4,5, and 8 which represents 195727301431 precisely 270390 times).

\section{Cross terms}

Every quadratic polynomial $f$ in $k$ variables (over $\mathbb{Q}$ ) can be written uniquely as $f(x)=Q(x)+\Lambda(x)+C$, where $Q(x)$ is a quadratic form in $k$ variables, $\Lambda(x)$ is a linear form, and $C$ is a constant. We will only consider quadratic polynomials such that $f(x) \in \mathbb{Z}$ for every $x \in \mathbb{Z}^{k}$. The quadratic form $Q(x)$ is positive definite if and only if $f(x)$ is bounded from below. As in the introduction, $f\left(x_{1}, x_{2}, \ldots, x_{k}\right)$ is a normalized totally positive quadratic polynomial if $f$ is quadratic, and the image of $\mathbb{Z}^{k}$ is contained in the nonnegative integers while it contains 0 . Clearly, for every positive definite quadratic form $Q(x)$ and linear form $\Lambda(x)$ there is a unique $C \in \mathbb{Z}$ such that $f(x)=Q(x)+\Lambda(x)+C$ is normalized totally positive. 
As noted before, $8 T_{x}=(2 x+1)^{2}-1=X^{2}-1$ if we put $X=2 x+1$. The polynomial $X^{2}-1$ is normalized totally positive on the odd integers. With $Y=$ $2 y+1$, we find $8 B_{x y}=4 x y+2 x+2 y=X Y-1$, where $B_{x y}:=\frac{1}{4}(2 x y+x+y)$ is the polynomial in $x, y$ satisfying $B_{x x}=T_{x}$. This way

$$
8\left(a T_{x}+b T_{y}+c B_{x y}\right)=a X^{2}+b Y^{2}+c X Y-(a+b+c) .
$$

If $C$ is the unique integer such that $a T_{x}+b T_{y}+c B_{x y}+C$ is normalized totally positive, then $a X^{2}+b Y^{2}+c X Y+(8 C-a-b-c)$ will be the corresponding shifted quadratic form that is normalized totally positive on the odd integers.

In order to describe our construction, we will say for simplicity that two quadratic polynomials $f_{1}$ and $f_{2}$ are (arithmetically) equivalent if the number of solutions to $f_{1}(x)=n$ equals the number of solutions to $f_{2}(x)=n$ for every integer $n \geq 0$.

We will consider a positive definite integral quadratic form (in $k$ variables) for which all cross terms in the matrix have even coefficients, so the cross terms of the quadratic form are $0 \bmod 4$. This restriction is natural if one keeps in mind that we are interested in the integers oddly represented by forms.

If $Q$ and $\widetilde{Q}$ are two equivalent quadratic forms such that the isomorphism preserves the condition that $X_{i}$ is odd, then we shall refer to them as equivalently odd and denote the equivalence class of such forms as $[Q]_{o}$.

For any positive definite quadratic form with cross terms divisible by four, we write

$$
Q=a_{1} X_{1}^{2}+\cdots+a_{k} X_{k}^{2}+\sum_{i \neq j} 4 c_{i j} X_{i} X_{j}
$$

We now define $f_{Q}=f_{[Q]_{o}}$ to be the unique normalized totally positive quadratic polynomial

$$
f_{Q}:=a_{1} T_{x_{1}}+\cdots+a_{k} T_{x_{k}}+\sum_{i \neq j} 4 c_{i j} B_{x_{i} x_{j}}+C .
$$

We will refer to $f_{Q}$ as a triangular sum with cross terms.

We will show that triangular sums with cross terms do not satisfy any finiteness theorem, and hence there is no overarching finiteness theorem for quadratic polynomials, as stated in Proposition 1.3. To do so, for every positive integer $n$ we will construct a triangular sum with cross terms $f_{n}$ which represents precisely every nonnegative integer other than $n$.

The following notation will be used. If $f$ and $g$ are polynomials in $k$ and $\ell$ variables, we denote by $f \oplus g$ the sum of the two as a polynomial in $k+\ell$ variables (so $f$ and $g$ are assumed to share no variables).

Theorem 3.1. Let $\mathcal{Z}$ be a subset of the positive integers. For every proper subset $\mathcal{Y} \subsetneq \mathcal{Z}$ there exists a triangular sum with cross terms representing $\mathcal{Y}$ but not representing $\mathcal{Z}$.

Proof. Let a proper subset $S_{0}$ of a given subset $S$ of the positive integers be given. Choose a positive integer $n \in S \backslash S_{0}$. We will proceed by explicit construction of the triangular sum with cross terms $f_{n}$ which represents every integer other than $n$.

First note that if the smallest positive integer not represented by $f$ is $n$, then, since the sum of three triangular numbers represents every nonnegative integer, we have that $f \oplus(n+1)\left(T_{x} \oplus T_{y} \oplus T_{z}\right)$ represents all $m \not \equiv n(\bmod n+1)$. But then we can choose $f_{n}:=f \oplus(n+1)\left(T_{x} \oplus T_{y} \oplus T_{z}\right) \oplus(2 n+1) T_{w}$. It is therefore equivalent to construct $f$ for which $n$ is the smallest positive integer not represented by $f$. 
Consider the quadratic form

$$
Q^{(N)}(X, Y):=N X^{2}+N Y^{2}+4 X Y
$$

and denote the corresponding triangular sum with cross terms by $f^{(N)}$; then

$$
f^{(N)}(x, y)=N T_{x}+N T_{y}+(2 x y+x+y)+1 .
$$

We first show that it is sufficient to determine that the generating function for $f^{(N)}$ is

$$
2+2 q+O\left(q^{N-12}\right) .
$$

Assuming equation (3.1), the generating function for $g_{n}:=\bigoplus_{i=1}^{n} f^{(N)}$ is

$$
2^{n}\left(1+\left(\begin{array}{l}
n \\
1
\end{array}\right) q+\cdots+\left(\begin{array}{l}
n \\
n
\end{array}\right) q^{n}\right)+O\left(q^{N-12}\right) .
$$

If we choose $N>n+13$, then the first integer not represented by $g$ is $n+1$. Therefore, we can take $f_{n}=g_{n-1}$; this also suffices for $n=1$ (if we interpret the empty direct sum $g_{0}$ as 0 ).

We now show that the generating function satisfies (3.1). Note that $f^{(N)}(0,-1)=$ $f^{(N)}(-1,0)=0$, while $f^{(N)}(0,0)=f^{(N)}(-1,-1)=1$. Now, without loss of generality, assume that $|x| \geq|y|$ and $x \notin\{0,-1\}$. Then,

$$
|2 x y+x+y| \leq 2|x|^{2}+2|x|=4 T_{|x|},
$$

so that

$$
f^{(N)}(x, y) \geq N T_{x}-4 T_{|x|}+N T_{y} .
$$

When $x \leq-2$ it is easy to check that $4 T_{|x|} \leq 12 T_{x}$ so that

$$
N T_{x}-4 T_{|x|} \geq(N-12) T_{x} \geq N-12
$$

and when $x>0$,

$$
N T_{x}-4 T_{|x|}=(N-4) T_{x} \geq N-4,
$$

since $T_{x} \geq 1$ for $x \notin\{0,-1\}$. Since $T_{y} \geq 0$, our assertion is verified.

It is important here to note how the above counterexamples differ from the proof when we only have diagonal terms, since this observation will lead us to the proof of Theorem 1.4 when $m_{f}$ is bounded.

Call a triangular sum with cross terms $f_{Q}$ (and also any corresponding $\widetilde{f_{Q}}$ ) a block if the corresponding quadratic form $Q$ has an irreducible matrix. We will build an escalator lattice by escalating (as a direct sum) by a block at each step. In Section 2, the breadth each time we escalated was finite, so that the overall tree was finite. In the above proof, however, there were infinitely many inequivalent blocks which represent 1 , so that the breadth is infinite. What was expressed in the above proof was that the supremum of these depths went to infinity as we chose $N$ increasing in terms of $n$ in the proof.

For

$$
f(x)=\sum_{i=1}^{k} b_{i} T_{x_{i}}+\sum_{1 \leq i<j \leq k} c_{i j}\left(2 x_{i} x_{j}+x_{i}+x_{j}\right)+C
$$

we will say that $f$ has (cross term) configuration $c=\left(c_{i j}\right)$. Since the matrix of $f$ is irreducible and hence the corresponding adjacency matrix is connected, we can assume throughout (by a change of variables) that for each $j>1$ there exists $i<j$ with $c_{i j} \neq 0$. 


\section{BOUNDED NORM}

We will now construct a natural norm on $f_{Q}$ such that restricting this norm will again give a finiteness result. Let a positive definite quadratic form with even cross terms in the corresponding matrix,

$$
Q(x):=\sum_{i=1}^{k} b_{i} x_{i}^{2}+\sum_{i<j} 4 c_{i j} x_{i} x_{j}
$$

be given. We define

$$
\widetilde{f}(x):=\widetilde{f_{Q}}(x):=\sum_{i=1}^{k} b_{i} T_{x_{i}}+\sum_{i<j, c_{i j} \geq 0} c_{i j}\left(2 x_{i} x_{j}+x_{i}+x_{j}\right)+\sum_{i<j, c_{i j}<0} c_{i j}\left(2 x_{i} x_{j}+x_{i}+x_{j}+1\right) .
$$

Remark 4.1. Note that the constant $c_{i j}$ is added every time $c_{i j}<0$; this may not seem canonical at first, but notice that if $Q^{\prime}$ is the equivalent quadratic form obtained by replacing $x_{1}$ with $-x_{1}$, then we find that this choice leads to $\widetilde{f_{Q}}=\widetilde{f_{Q^{\prime}}}$.

We next define

$$
\widetilde{m}_{\tilde{f}}:=-\min _{x \in \mathbb{Z}^{k}} \widetilde{f}(x),
$$

which is added to obtain the unique (up to equivalence) normalized totally positive quadratic polynomial $f_{Q}=\widetilde{f_{Q}}+\widetilde{m}_{\widetilde{f}}$ corresponding to $Q$. Thus, we can define the norm

$$
m_{f_{Q}}:=m_{[Q]_{o}}:=\min _{Q^{\prime} \in[Q]_{o}}\left|\widetilde{m}_{\widetilde{f_{Q^{\prime}}}}\right| .
$$

In a sense, this norm measures the distance between $f_{Q}$ and the closest $\widetilde{f_{Q^{\prime}}}$ in the equivalence class, where the distance is merely given by the absolute value of the normalization factor required. If $m_{f}$ is bounded, then we will again find that checking a finite subset will suffice. We may now state the following more precise version of Theorem 1.4.

Theorem 4.2. Fix an integer $m$ and a subset $\mathcal{Z}$ of the positive integers. Then there is a finite subset $\mathcal{Y}_{m} \subset \mathcal{Z}$ depending only on $m$ and $\mathcal{Z}$ such that every triangular sum with cross terms $f$ satisfying $m_{f} \leq m$ represents $\mathcal{Z}$ if and only if it represents $\mathcal{Y}_{m}$.

Moreover, for $\mathcal{Z}$ equal to the positive integers, we find $\max \mathcal{Y}_{m} \gg m^{2}$.

Remark 4.3. It may be of interest to investigate the growth of $\max \mathcal{Y}_{m}$ in terms of $m$ in the case where $\mathcal{Z}$ consists of all positive integers. The $m=0$ case is precisely Theorem 1.1. Following the bounds given in the proof of Theorem 1.4. computational evidence suggests that $\mathcal{Y}_{1}\left(\mathbb{Z}_{>0}\right)$ equals

$$
\begin{aligned}
& \{1,2,3,4,5,6,8,9,10,11,12,13,14,16,17,19,20,23,24,25,26,29,32,33,34,35, \\
& 38,41,46,47,48,50,53,54,58,62,63,75,86,96,101,102,113,117,129,162,195,
\end{aligned}
$$

A proof of the above identity using the techniques of Bhargava and Hanke 2 . developed in the proof of the 290-Theorem may require a careful analysis of a possible Siegel zero. To exhibit this difficulty, consider the sum $g(x, y, z)=T_{x}+$ $2 T_{y}+6 T_{z}$. In the construction of $\mathcal{Y}_{1}\left(\mathbb{Z}_{>0}\right)$ the computations imply that there are infinitely many $Q$ with $m_{f_{Q}}=1$ for which $g \oplus f_{Q}$ represents every positive integer. 
Hence we cannot merely check each case individually and must know information about the integers represented by $g$ independently.

Although it seems that $g$ represents all odd integers, a proof of this appears to be beyond current techniques due to ineffective lower bounds for the class number (see [10]). However, since a possible Siegel zero for $L\left(\chi_{d}, s\right)$ would give a lower bound for the class number when $d^{\prime} \neq d$ (both fundamental), one may be able to show that $g$ represents at least one of $n$ or $n-1$ for every positive integer $n$, which would suffice for showing the above identity.

We will first give an overview of the proof; details can be found in the next section.

Fix a positive integer $m$. As in the above remark, we will escalate with blocks. We will first show that when $m_{f} \leq m$, the number of blocks that are not dimension 1 in any branch of the escalator tree is bounded and that there are only finitely many choices for the configuration of each block. We will then proceed by defining $N\left(M_{1}, M_{2}, \ldots, M_{k}, c\right)$ to be the smallest integer not represented by the totally positive quadratic polynomial corresponding to

$\widetilde{f}(x):=\sum_{i=1}^{k} M_{i} T_{x_{i}}+\sum_{i<j, c_{i j} \geq 0} c_{i j}\left(2 x_{i} x_{j}+x_{i}+x_{j}\right)+\sum_{i<j, c_{i j}<0} c_{i j}\left(2 x_{i} x_{j}+x_{i}+x_{j}+1\right)$.

Our claim is then equivalent to showing that in the escalator tree,

$$
\sup _{M_{1}, \ldots, M_{k}, c} N\left(M_{1}, M_{2}, \ldots, M_{k}, c\right)
$$

is finite. To do so, we will effectively show that with the configurations of blocks of dimension greater than one fixed, the supremum with $M_{i}$ sufficiently large is finite and independent of the choice of $M_{i}$, and then fix $M_{1} \leq m_{1}$, and again show that the resulting supremum is independent of $M_{2}, \ldots, M_{k}$, and so forth. Since there are only finitely many such choices of $c$, the result comes from taking the maximum of each of these suprema.

\section{ProOF}

To prove Theorem 4.2, and hence Theorem 1.4, we begin with a lemma that will show that there are only finitely many choices of the cross term configuration.

Lemma 5.1. If $m_{f} \leq m$, then there are only finitely many choices of the cross term configurations $c_{i j}$ of all blocks of dimension greater than one, up to equivalent forms.

Proof. First note that $m_{f \oplus g}=m_{f}+m_{g}$, so that we can only have at most $m$ blocks $f$ with $m_{f}>0$, while we will see that $m_{f}>0$ unless $f$ is one dimensional (and hence the block is a constant times $T_{x}$ ). It therefore suffices to show that each block $f$ of dimension greater than one has $m_{f}>0$ and those with the restriction $m_{f} \leq m$ have bounded dimension and bounded coefficients in the configuration. Fix the configuration $c$ of a block $\widetilde{f}$ with dimension $k$ such that $\widetilde{m}_{\tilde{f}}=m_{f}$, namely a minimal element. We will recursively show a particular choice of $x_{i}$ such that

$$
\tilde{f}(x) \leq-\max \left\{\max _{i, j}\left|c_{i j}\right|, k-1\right\},
$$

so that the max of the $c_{i j}$ is bounded by $m$, and the dimension is bounded by $m+1$. 
First set $x_{1}=0$. Since $\tilde{f}$ is a block, we know at step $j$ that there is some $i<j$ such that $c_{i j} \neq 0$. Choose $i<j$ such that $\left|c_{i j}\right|$ is maximal. If $x_{i}=0$, then we set $x_{j}=-1$ if $c_{i j}>0$ and $x_{j}=0$ otherwise. If $x_{i}=-1$, then we set $x_{j}=0$ if $c_{i j}>0$ and $x_{j}=-1$ otherwise.

Since all of our choices of $x_{i}$ are 0 or -1 and $T_{-1}=T_{0}=0$, the integer represented is independent of the diagonal terms $M_{i}$. Now we note that for $x_{i}, x_{j} \in\{0,-1\}$ we have $2 x_{i} x_{j}+x_{i}+x_{j}=0$ if $x_{i}=x_{j}$ and $2 x_{i} x_{j}+x_{i}+x_{j}=-1$ otherwise. Therefore, if $x_{i}=x_{j}$, then from our definition of $\tilde{f}$, the cross term corresponding to $c_{i j}$ adds 0 if $c_{i j} \geq 0$ and adds $-\left|c_{i j}\right|$ otherwise. If $x_{i}=0$ and $x_{j}=-1$, then the cross term adds $-\left|c_{i j}\right|$ if $c_{i j} \geq 0$ and adds 0 otherwise. Therefore by our construction above, we know that for $\left|c_{i j}\right|$ maximal, we have added $-\left|c_{i j}\right|$ to our sum, and we never add a positive integer, so the sum is at most $-\left|c_{i j}\right|$. Moreover, since the block is connected, we have added at most -1 at each inductive step, so that the sum is at most $-(k-1)$.

For simplicity, in our escalator tree, we will "push" up all of the blocks to the top of the tree which are not dimension 1. To do so, we will first build the tree with all possible choices of blocks which are not dimension 1 and then escalate with only dimension 1 blocks from each of the nodes of the tree, including the root (the empty set). Thus, every possible form will show up in our representation. This tree (without the blocks of dimension 1) is depth at most $m$ in the number of blocks, but is of infinite breadth. Henceforth, we can consider the configuration $c$ to be fixed, and take the maximum over all choices of $c$.

We will now see that the subtree from each fixed node is of finite depth. Consider the corresponding quadratic form $Q$. First note that the generating function for $Q$ when all $x_{i}$ are odd is the generating function for $Q$ minus the generating function with some $x_{i}$ even, and the others arbitrary, which is simply the generating function for another quadratic form without any restrictions, taking $x_{i} \rightarrow 2 x_{i}$. Thus, we have the difference of $\theta$-series for finitely many quadratic forms, and hence the Fourier expansion is a modular form. Now we simply note that any modular form can be decomposed into an Eisenstein series and a cusp form (cf. [13]). Using the bounds of Tartakowsky [16] and Deligne [6], as long as the Eisenstein series is nonzero, the growth of the coefficients of the Eisenstein series can be shown to grow more quickly than the coefficients of the cusp form whenever the dimension is greater than or equal to 5 , other than finitely many congruence classes for which the coefficients of both the Eisenstein series and the cusp form are zero.

Therefore, as long as the Eisenstein series is nonzero, there are only finitely many congruence classes and finitely many "sporadic" integers which are not represented by the quadratic form. Thus, after dimension 5, there are only finitely many congruence classes and finitely many sporadic integers not represented by the form $f$. If at any step of the escalation, any of the integers in these congruence classes is represented, then we have fewer congruence classes, and only finitely many more sporadic integers which are not represented, so that the resulting depth is bounded. For the dimension 1 blocks, it is clear that the breadth of each escalation is finite, so there are only finitely many escalators coming from this node. Therefore, it suffices to show that the Eisenstein series is nonzero.

Again using Siegel's theorem [14, the coefficients of the Eisenstein series are simply a linear combination of the values given by the local densities of the quadratic 
forms from the above linear combination of $\theta$-series. At every prime other than $p=$ 2 , the local densities of the quadratic forms, of which we are taking the difference of $\theta$-series, are equal, so we only need to show that the difference of the local densities at $p=2$ is positive. However, the difference of the number of local representations at a fixed 2-power must be positive, since the integer is locally represented with $x_{i}$ odd, except possibly for finitely many congruence classes if a high 2-power divides the discriminant.

Therefore, we can define $\tilde{N}\left(M_{1}, \ldots, M_{k}, c\right)$ to be the maximum of $N\left(M_{1}, \ldots\right.$, $\left.M_{k}, M_{k+1}, \ldots, M_{l}, c\right)$, where $M_{k+1}$ to $M_{l}$ are the dimension 1 blocks coming from the (finite) subtree of this node. We will show that $\widetilde{N}\left(M_{1}, \ldots, M_{k}, c\right)$ is independent of the choice of $M_{i}$ whenever $M_{i}$ is sufficiently large by showing that the resulting subtrees are identical. We need the following lemma to obtain this goal. We will need some notation before we proceed.

For a set $T$, define $q^{T}:=\sum_{t \in T} q^{t}$, a formal power series in $q$. For fixed sets $S, T \subseteq \mathbb{N}$, we will say that a form $f(x):=\sum b_{i} T_{x_{i}}$ represents $S / T$ if for every $s \in S$ the coefficient of $q^{s}$ in $q^{T} g(q)$ is positive, where $g(q)$ is the generating function for $f(x)$ given by $g(q):=\sum_{x \in \mathbb{Z}^{k}} q^{f(x)}$.

Lemma 5.2. Let a (diagonal) triangular form $f$ be given. Fix $S, T_{1}, T_{2} \subseteq \mathbb{N}$ and $M \in \mathbb{N}$ such that $\min _{n \in T_{2}} \geq M$. Define $T:=T_{1} \cup T_{2}$. Then there exists a bound $M_{T_{1}, S}$ and a finite subset $S_{0} \subseteq S$, depending only on $T_{1}$ and $S$ such that if $M>M_{T_{1}, S}$, then $f$ represents $S / T$ if and only if $f$ represents $S_{0} / T_{1}$.

Proof. We will escalate as in [1] with a slight deviation. At each escalation node, there is a least element $s \in S$ such that $S / T_{1}$ is not represented by the form $f$ corresponding to this node. As in [1, we shall refer to $s$ as the truant of $f$. To represent $\{s\} / T_{1}$, we must have some $t_{1} \in T_{1}$ such that $s-t_{1}$ is represented by $f+b T_{x}$. Therefore, for each $t_{1}<s$ we escalate with finitely many choices of $b$, and there are only finitely many choices of $t_{1}$. Thus, the breadth at each escalation is finite, and our argument above using modular forms shows that the depth is also finite, so there are only finitely many choices of $s \in S$ which are truants in the escalation tree. Take $S_{0}$ to be the set of truants in the escalation tree and define $M_{T_{1}, S}:=\max s \in S_{0} s+1$. The argument above shows that representing $S / T_{1}$ is equivalent to representing $S_{0} / T_{1}$. When following the above process with $T$ instead of $T_{1}$ whenever $M>M_{T_{1}, S}$, we will have the same subtree and the same truants at each step, so that representing $S / T$ is equivalent to representing $S / T_{1}$, and hence representing $S / T$ is equivalent to representing $S_{0} / T_{1}$.

Remark 5.3. It is of interest to note that if we replace "(diagonal) triangular form" with "quadratic form" (without the odd condition), the proof follows verbatim, since the breadth is also finite, so that this can be considered a generalization of Bhargava's result that there is always a finite subset $S_{0}$ of $S$ such that the quadratic form represents $S$ if and only if it represents $S_{0}$, since this is obtained by taking $T_{1}=T=\{0\}$.

Now consider $X_{j}:=\left\{x: x_{i}\right.$ arbitrary for $i \leq j, x_{i} \in\{0,-1\}$ otherwise $\}$ and define $T_{1, j}:=\left\{f(x): x \in X_{j}\right\}$ and $T_{2, j}:=\left\{f(x): x \notin X_{j}\right\}$. We will use Lemma 5.2 with $T_{1}=T_{1, j}$ and $T_{2}=T_{2, j}$ for each $0 \leq j \leq k$. To use the lemma effectively, we will show the following lemma. 
Lemma 5.4. There exist bounds $M_{X_{j}}^{(i)}$ depending only on $M_{1}, \ldots, M_{j}, c$ such that if $M_{i} \geq M_{X_{j}}^{(i)}$ for every $i>j$, then the smallest element of $T_{2, j}$ is greater than $M_{T_{1}, \mathbb{N}}$, where $M_{T_{1}, \mathbb{N}}$ is as defined in Lemma 5.2 .

Proof. We will proceed by induction. For $j=0$, we will take $M_{X_{0}}^{(i)}=M_{T_{1,0}, \mathbb{N}}+$ $6 \sum_{j}\left|c_{i j}\right|$. Noting that for $\left|x_{j}\right|<\left|x_{i}\right|$ we have $\left|2\left(x_{i}-\frac{x_{j}}{2}\right) x_{j}\right| \leq x_{i}^{2}$, we get the inequality

$$
c_{i j}\left(2 x_{i} x_{j}+x_{i}+x_{j}\right) \geq-\left|c_{i j}\right|\left(2 T_{\left|x_{i}\right|}+2 T_{\left|x_{j}\right|}\right) .
$$

The case $j=0$ then follows from the fact that for $x_{i} \notin\{0,-1\}$ we have $T_{\left|x_{i}\right|} \leq 3 T_{x_{i}}$.

We now continue by induction on $j$. For the corresponding quadratic form, we note that plugging in $x_{1}=\frac{-\sum_{j>1} c_{1 j} x_{j}}{2 M_{1}}$ gives the minimal value over the reals. The quadratic form $Q^{\prime}$ obtained by specializing this value of $x_{1}$ has rational coefficients with denominator dividing $2 M_{1}$. We therefore can consider $\widetilde{Q}:=4 M_{1} \cdot Q^{\prime}$, which is a quadratic form of the desired type. Thus, we can use the inductive step for $\widetilde{Q}$. But this gives a bound which minimizes $\widetilde{Q}$, and hence $Q^{\prime}$, but an arbitrary choice of $x_{1}$ must give a value greater than or equal to this, so the result follows.

Now, by our choice of $X_{j}, T_{1, j}$ is independent of $M_{i}$ for $i>j$, since $T_{x_{i}}=0$. Thus, fix $c$ and take $M_{i} \geq M_{X_{0}}^{(i)}$. Then the corresponding subtrees are independent of the choice of $M_{i}$, so that $\sup \widetilde{N}\left(M_{1}, \ldots, M_{k}, c\right)$ is the unique largest truant in the subtree (effectively we may replace $M_{i}=\infty$ ). We may now fix $M_{1} \leq M_{X_{0}}^{(1)}$, since there are only finitely many such choices. With this $M_{1}$ fixed, we define $T_{1,1}$ as above, and again find bounds for the other $M_{i}$. Continuing recursively gives the desired result, since we know that $k \leq m$, so there are only finitely many suprema that we take.

To show that $\max \mathcal{Y}_{m}\left(\mathbb{Z}_{>0}\right) \gg m^{2}$, we consider again the construction of our counterexamples. Consider $f(x, y):=\bigoplus_{i=1}^{m} f^{(N)} \oplus T_{y}$. Since $T_{r}=\sum_{n=1}^{r} n$, for $N$ sufficiently large the smallest integer not represented by $f$ is clearly $T_{m+1}-1 \gg m^{2}$.

\section{ACKNOWLEDGEMENTS}

The authors would like to thank W.K. Chan for helpful comments, as well as the anonymous referee for a detailed and helpful report.

\section{REFERENCES}

[1] M. Bhargava, On the Conway-Schneeberger fifteen theorem, Quadratic forms and their applications (Dublin, 1999), Contemp. Math. 272, Amer. Math. Soc., 2000, 27-37. MR1803359 (2001m:11050)

[2] M. Bhargava, J. Hanke, Universal quadratic forms and the 290-Theorem, Invent. Math., to appear.

[3] A. Cauchy, Démonstration du théorème général de Fermat sur les nombres polygones, Mém. Sci. Math. Phys. Inst. France 14 (1813-1815), 177-220; Oeuvres complètes VI (1905), 320-353.

[4] J.H. Conway, Universal quadratic forms and the fifteen theorem, Contemp. Math. 272, Amer. Math. Soc., 2000, 23-26. MR1803358(2001m:11049)

[5] D. Cox, Primes of the form $x^{2}+n y^{2}$, New York: Wiley, 1989. MR1028322 (90m:11016)

[6] P. Deligne, La Conjecture de Weil I, Inst. Hautes Études Sci. Publ. Math. 43 (1974), 273-307. MR0340258(49:5013)

[7] L.E. Dickson, Quaternary quadratic forms representing all integers, Amer. J. Math. 49 (1927), 39-56. MR 1506600 
[8] W. Duke, Some old problems and new results about quadratic forms, Notices Amer. Math. Soc. 44 (1997), 190-196. MR 1426107 (97i:11035)

[9] B. Jones, The arithmetic theory of quadratic forms, Carcus Monograph Series 10, Math. Assoc. Amer., Buffalo, NY, 1950. MR0037321(12:244a)

[10] B. Kane, Representing sets with sums of triangular numbers, Int. Math. Res. Not. 2009 (2009), 3264-3285. MR2534998 (2011c:11055)

[11] J. Liouville, Nouveaux théorèmes concernant les nombres triangulaires, Journal de Mathématiques Pures et Appliquées 8 (1863), 73-84.

[12] M.B. Nathanson, A short proof of Cauchy's polygonal number theorem, Proc. Amer. Math. Soc. 99 (1987), 22-24. MR866422 (88d:11023)

[13] K. Ono, Web of Modularity: Arithmetic of the coefficients of modular forms and q-series, Amer. Math. Soc., Providence, RI, 2004. MR2020489 (2005c:11053)

[14] C. Siegel, Über die analytische Theorie der quadratischen Formen, Ann. of Math. (2) 36 (1935), 527-606. MR1503238

[15] J. Sturm, On the congruence of modular forms, in: Number theory (New York, 1984-1985), Springer, Berlin, 1987, 275-280. MR894516 (88h:11031)

[16] W. Tartakowsky, Die Gesamtheit der Zahlen, die durch eine quadratische Form $F\left(x_{1}, x_{2}, \ldots, x_{s}\right),(s \geq 4)$ darstellbar sind, Izv. Akad. Nauk SSSR 7 (1929), 111-122, 165-196.

Radboud Universiteit, Heijendaalseweg 135, 6525 AJ Nijmegen, The Netherlands

E-mail address: bosma@math.ru.nl

Radboud Universiteit, Heijendaalseweg 135, 6525 AJ Nijmegen, The Netherlands

Current address: Mathematical Institute, University of Cologne, Weyertal 86-90, 50931 Cologne, Germany

E-mail address: bkane@mi.uni-koeln.de 